\title{
Impact du mode de désherbage de l'oignon sur sa productivité et la qualité des bulbes au centre du Burkina Faso
}

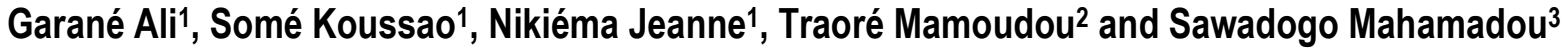 \\ ${ }^{1^{*}}$ Institut de l'Environnement et de Recherches Agricoles (INERA/CREAF-Kamboinse), Département Production \\ Végétale/Programme Cultures Maraîchères, Fruitières, Plantes à Tubercules, 01 BP 470 Ouagadougou 01, Burkina \\ Faso. \\ 2Institut de l'Environnement et de Recherches Agricoles (INERA/CREAF-Kamboinsè), Département Gestion des \\ Ressources Naturelles/Système de Production, 03 BP 470 Ouagadougou 03, Burkina Faso. \\ 3Université de Ouaga I Pr Joseph Ki -Zerbo/Unité de Formation et de Recherche en Science de la Vie et de la Terre \\ (UO/UFR-SVT), Laboratoire de Génétique et Biotechnologie Végétale, 03 BP 7021 Ouagadougou 03, Burkina Faso. \\ *Correspondance, courriel: ali_garane@yahoo.fr
}

Original submitted in on $18^{\text {th }}$ April 2019. Published online at www.m.elewa.org/journals/ on $31^{\text {st }}$ July 2019 https://dx.doi.org/10.4314/jab.v139i1.5

\section{RESUME}

Objectif: Cette étude vise à apprécier au centre du Burkina Faso l'effet du mode de désherbage sur la productivité de l'oignon et la qualité des bulbes récoltés afin de proposer une méthode permettant un meilleur contrôle de l'enherbement de cette culture dans la zone.

Méthodologie et résultats: Le dispositif expérimental est un bloc de Fisher à 4 répétitions et 6 traitements constitués des formulations $\mathrm{T} 1, \mathrm{~T} 2, \mathrm{~T} 3, \mathrm{~T} 4, \mathrm{~T} 5$ et $\mathrm{T} 6$. Les résultats ont montré que le sarclage manuel au 30 ème et $60^{\mathrm{ème}}$ jour après repiquage (T3) et la Pendimethaline (herbicide) appliquée en pré-levée uniquement (T4) ou suivie d'un sarclage manuel au 60ème jour après repiquage (T6) ont occasionné les plus grands nombres de bulbes respectifs de 116,$75 ; 117,5$ et 120,25 bulbes $/ 6 \mathrm{~m}^{2}$. Le poids moyen bulbe a

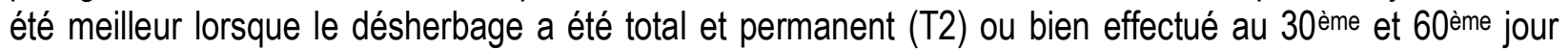
après repiquage avec 41,55 et $26,3 \mathrm{~g}$ respectivement. Par rapport à l'ensemble des traitements, le désherbage total et permanent et dans une moindre mesure, le sarclage manuel effectué au 30 ème et 60 ème jour après repiquage ont observé des rendements bulbes quelques peu meilleurs respectifs de $4,13 \mathrm{~kg}$ et $3,17 \mathrm{~kg} / 6 \mathrm{~m}^{2}$. L'absence de désherbage (T1) et la Clethodim (herbicide) en post-levée (T5) ont entrainé une plus faible masse de bulbes à la récolte. Indépendamment des traitements appliqués, la matière sèche du bulbe a très peu varié allant de 11,72\% (T6) à 12,8\% (T1).

Conclusion et application des résultats: Le désherbage continu et permanent a observé un nombre minimum de 10,5 bulbes $/ 6 \mathrm{~m}^{2}$ de petits calibres et la Clethodim en post-levée un maximum de 26,5 bulbes $/ 6 \mathrm{~m}^{2}$ de petits calibres. Une même tendance a été observée pour le poids des bulbes de petits calibres, le désherbage continu et permanent a conservé la plus faible valeur de $0,04 \mathrm{~kg} / 6 \mathrm{~m}^{2}$. Par contre, le sarclage manuel au 30 ème et 60 ème jour après repiquage $\left(0,08 \mathrm{~kg} / 6 \mathrm{~m}^{2}\right)$, la Pendimethaline en pré-levée et la Clethodim en post-levée ont observé la plus forte valeur de $0,09 \mathrm{Kg} / 6 \mathrm{~m}^{2}$ de bulbes démontrant une performance moindre de ces deux molécules appliquées. Les sarclo-binages fréquents en cultures maraîchères exigent une main-d'œuvre importante et un nombre élevé d'heure de travail. Leur association 
avec des herbicides sélectifs (T6) reste envisageable dans la gestion des mauvaises herbes sur les grandes superficies pour une réduction des coûts d'exploitation.

Mots clés: adventices, oignon, herbicides, sarclage, centre, Burkina Faso

\section{Impact of weed control on the productivity and quality of onion bulbs in the center of Burkina Faso ABSTRACT}

Objective: This study aims to assess in central Burkina Faso the effect of the method of weeding on the productivity of the onion and the quality of the harvested bulbs in order to propose a method allowing a better control of the weed cover of this crop in the zone.

Methodology and results: The experimental design is a randomized complet block with 4 replications and 6 treatments with the following formulations T1, T2, T3, T4, T5 and T6. The results showed that manual weeding on the 30th and 60th day after transplanting (T3) and Pendimethalin (herbicide) applied in preemergence only (T4) or followed by Manual Weeding on the 60th day after transplanting (T6) resulted in the largest numbers of respective bulbs of $116.75 ; 117.5$ and 120.25 bulbs / $6 \mathrm{~m}^{2}$. The average bulb weight transplanting with 41.55 and $26.3 \mathrm{~g}$ respectively. With respect to all treatments, total and permanent weeding and also manual weeding carried out on the 30th and 60th day after transplanting, have observed slightly better bulbs yields of $4.13 \mathrm{~kg}$ and $3.17 \mathrm{~kg} / 6 \mathrm{~m}^{2}$ respectively. The absence of weeding (Control) and Clethodim (herbicide) in post-emergence (T5) resulted in a lower yield at harvest. Regardless of the applied treatments, the dry matter of the bulb has shown a very low variability ranging from $11.72 \%$ (T6) to $12.8 \%$ (T1).

Conclusion and application of results Continuous and permanent weeding have observed a minimum number of 10.5 bulbs / $6 \mathrm{~m}^{2}$ of small caliber and Clethodim in post-emergence a maximum of 26.5 bulbs / 6 $\mathrm{m}^{2}$ of small caliber. The same trend was observed for the weight of small-caliber bulbs, with the continuous and permanent weeding which kept the lowest value of $0.04 \mathrm{~kg} / 6 \mathrm{~m}^{2}$. On the other hand, the Manual Weeding on the 30th and 60th day after transplanting $\left(0.08 \mathrm{~kg} / 6 \mathrm{~m}^{2}\right)$, the Pendimethalin in pre-emergence and the Clethodim in post-emergence have observed the highest value of $0.09 \mathrm{~kg} / 6 \mathrm{~m}^{2}$ of bulbs demonstrating a lower performance of these two molecules applied. Weeding and frequent hoeing in vegetable crops require an important workforce and is demanding in time. Their association with selective herbicides (T6) remains possible in the weed control on large areas for a reduction of operating costs.

Keywords: Weeds, onion, herbicides, weeding, center, Burkina Faso

\section{INTRODUCTION}

L'oignon constitue la principale culture maraîchère du Burkina Faso après la tomate. La production totale de l'oignon bulbe était de 242258 tonnes, soit $32,4 \%$ de la production totale maraîchère (DPSAA, 2011). Les rendements ont évolué de 15 tha en 2005 à 21 t/ha en 2008, soit une progression d'environ 40\% (DPSAA, 2011). La culture d'oignon est sujette à de nombreuses difficultés dont entre autres les problèmes phytosanitaires parmi lesquels les mauvaises herbes jouent un rôle prépondérant. En effet, la faible couverture du sol et la lenteur de croissance caractéristique en début de cycle rend l'oignon plus sensible à la compétition des mauvaises herbes (Carlson and Kirby, 2005; Qasem, 2006;
Smith et al., 2008; Mario, 2015). Le sarclage manuel reste la méthode de lutte la plus courante employée au détriment du contrôle chimique pour le contrôle mauvaises herbes dans les parcelles d'oignon (Bello et al., 2004; Alessandro et Alseny, 2008; Son et al., 2016). Pour réduire voire annihiler la compétition précoce des adventices et lutter contre les espèces plus résistantes, difficiles a éradiquer, il est primordial de trouver des méthodes améliorées de lutte avec un recoure au traitement chimique (Hodonou et al., 2001; Bello et al., 2012; Mario, 2015). Le présent travail envisage d'étudier l'effet du mode de désherbage manuel et chimique de l'oignon sur sa productivité et la qualité des bulbes au centre du Burkina Faso. 


\section{MATERIEL ET METHODES}

Brève description du milieu biogéographique: Le CREAF est basé à kamboinsé, localité située à une douzaine de kilomètres au Nord de Ouagadougou sur l'axe Ouaga-Kongoussi. Le CREAF reçoit une pluviométrie annuelle de $700 \mathrm{~mm}$. Ainsi, la zone agroécologique à laquelle il appartient est le secteur nord-soudanien situé entre les latitudes $13^{\circ} \mathrm{N}$ et $11^{\circ} 30$ $\mathrm{N}$ avec des précipitations annuelles maximales de 750 à $1000 \mathrm{~mm}$ (Thiombiano, 2005). II couvre les localités de Fada N'Gourma, Tenkodogo, Ouagadougou, Dédougou et Tougan. Les sols sont de types ferrugineux remaniés et sont, de par leur situation topographique très sensible à l'érosion hydrique. C'est la zone par excellence de tous les types de savanes avec une légère dominance des savanes arbustives et arborées. Le tapis herbacé est surtout formé de plusieurs espèces de dicotylédones et de graminées. Parmi les graminées vivaces citons Imperata cylindrica, auxquelles s'ajoutent plusieurs espèces de cypéracée, la strate arborée est composée d'essences dépassant rarement $20 \mathrm{~m}$ de haut.

Matériel expérimental: Matériel végétal utilisé est constitué par la variété d'oignon Violet de galmi de 90 jours de précocité. Le rendement potentiel varie entre 30 et 80 t/ha.

Les engrais NPK (14-23-14), l'urée (46\%) ainsi que du fumier d'étable bien décomposé ont été utilisés. L'herbicide Alligator 400 EC (Pendimethaline $400 \mathrm{~g} / \mathrm{l}$ ) a été utilisé en pré-levée à la dose $2 \mathrm{~L} /$ ha et le Select 120 EC (Clethodim $120 \mathrm{~g} / \mathrm{l}$ ) en post-levée à la dose 1L/ha.

Dispositif expérimental: Le dispositif expérimental utilisé est un bloc Fisher (complètement randomisé) constitué de quatre répétitions et de six traitements ( $\mathrm{T} 1$, T2, T3, T4, T5, T6). La parcelle élémentaire est une planche de $4,0 \mathrm{~m}$ de long sur $1,5 \mathrm{~m}$ de large soit $\left(6 \mathrm{~m}^{2}\right)$ de surface par traitement au nombre de $24(6 \times 4$ blocs). L'écart est de 0,5 $\mathrm{m}$ entre les planches et de 1 $\mathrm{m}$ entre les blocs au nombre de 4. La superficie totale est de $238,5 \mathrm{~m}^{2}(26,5 \mathrm{~m} \times 9 \mathrm{~m})$ pour une superficie utile de $144 \mathrm{~m}^{2}$ (24 m x $\left.6 \mathrm{~m}\right)$. Les plants d'oignon ont été repiqués suivants les écartements de $20 \mathrm{~cm}$ entre les lignes et $15 \mathrm{~cm}$ entre les plants $(20 \mathrm{~cm} \times 15 \mathrm{~cm})$. Au repiquage, la densité est de 333333 plants à l'hectare ou de 200 plants par planche de $6 \mathrm{~m}^{2}$ de surface. La figure 1 schématise le dispositif de l'essai. Des traitements ont été appliqués à chaque parcelle élémentaire (Tableau 1).

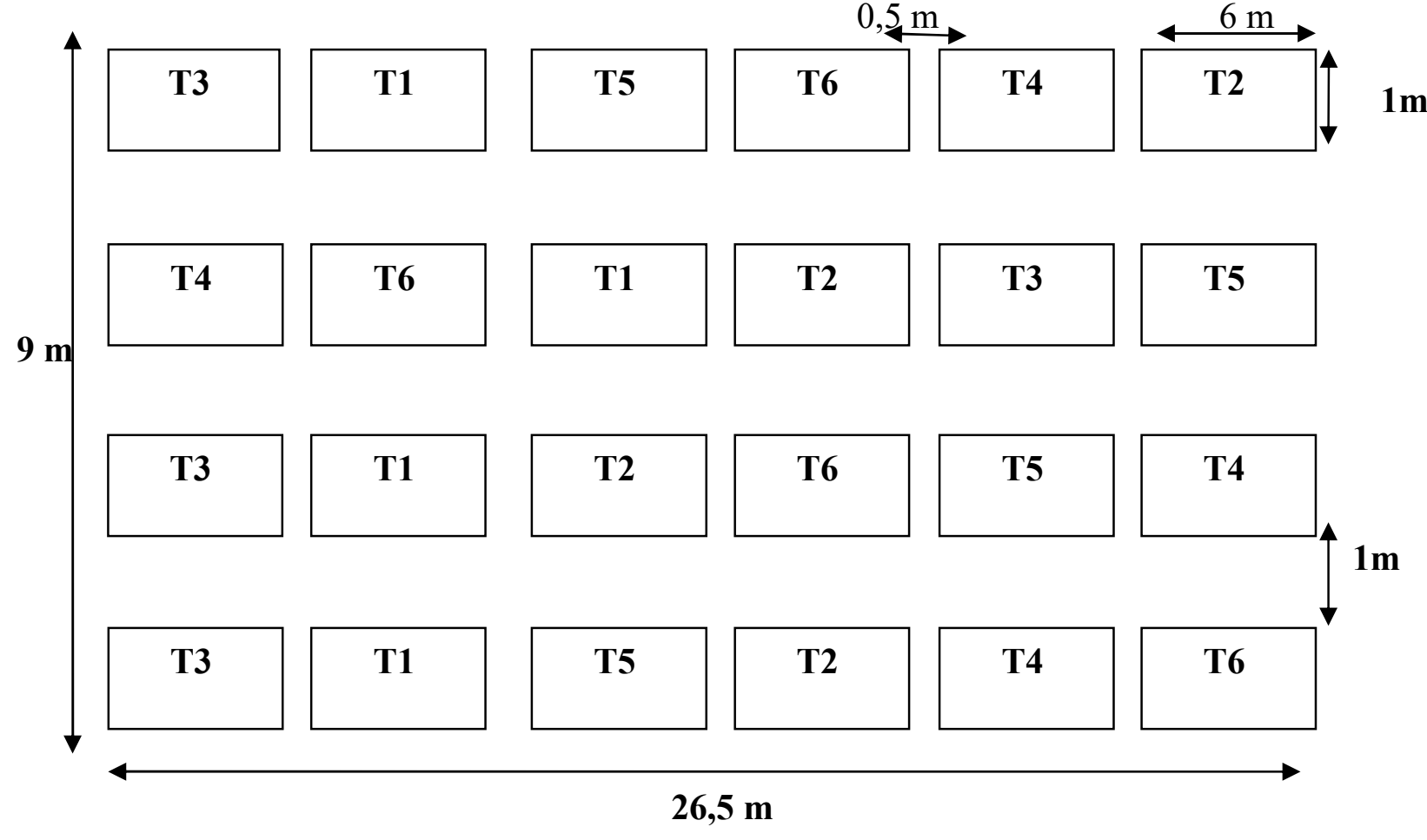

Figure 1: Dispositif expérimental utilisé 
Tableau 1: Traitements appliqués aux expérimentations et leur formulation

\begin{tabular}{l|l}
\hline \multicolumn{1}{c|}{ Traitements } & \multicolumn{1}{c}{ Formulation } \\
\hline T1 (parcelle témoin) & Les parcelles n'ont reçu aucun désherbage chimique ne manuel \\
T2 (témoin de référence) & Désherbage total et permanent \\
T3 & SM (Sarclage manuel) au 30ème et 60ème jour après repiquage \\
T4 & $\begin{array}{l}\text { L'herbicide Alligator (Pendimethaline) appliqué en pré-levée) à la dose de } 2 \mathrm{~L} \\
\text { par hectare }\end{array}$ \\
T5 & L'herbicide Select (Clethodime) appliqué en post-levée à la dose de $1 \mathrm{~L}$ par \\
T6 & hectare \\
& L'herbicide Alligator (Pendimethaline) est appliqué en pré-levée) à la dose de 2 \\
\hline
\end{tabular}

Conduite de l'essai: Les essais ont été conduits sur des parcelles expérimentales du Centre National de Recherches Environnementales et Agricole de Kamboinsè (CREAF-K) de l'INERA en 2015 et 2016. La variété est semée en pépinière le 5 décembre en terre désinfectée au préalable à la chaleur. La levée est générale 15 jours après semis soit le $20 / 12 / 2015$. Les plantules d'oignon ont été repiquées le 17 janvier 2016 soit environ 42 jours après le semis. Les applications de l'herbicide de pré-levée Alligator $400 \mathrm{EC}$, matière active la Pendimethaline $400 \mathrm{~g} / \mathrm{l}$ dose $2 \mathrm{~L} / \mathrm{ha}$ et de I'herbicide de post-levée Seclect 120 EC ayant la Clethodim $120 \mathrm{~g} / \mathrm{l}$ comme matière active dose $1 \mathrm{~L} / \mathrm{ha}$ ont été effectuées le 18 décembre 2015 et le 8 janvier 2016 respectivement. Le premier et le deuxième sarclage ont été réalisés respectivement le 17 janvier et 17 février 2016, soit le 30 ème et 60 ème JAR dans le traitement 3. Quatre sarclo-binages (15 et $30 \mathrm{JAR}$, à $45 \mathrm{JAR}$ et à $60 \mathrm{JAR}$ ) ont été indispensables dans le témoin de référence (T2). Le Décis a été utilisé comme insecticide en cours de végétation. La fumure de fonds composée de 20 tha de fumier, soit $12 \mathrm{~kg}$ de fumiers bien décomposé par planche de $6 \mathrm{~m}^{2}$; également, 350 $\mathrm{kg} / \mathrm{ha}$ de NPK, soit $208 \mathrm{~g}$ par planche de $6 \mathrm{~m}^{2}$ est apportée au cours de la préparation du sol. La fumure

\section{RESULTATS}

Effets du traitement sur le nombre de bulbes récoltés: Le nombre total de bulbes récoltés est présenté dans le Tableau 2. Les traitements appliqués ont été par ce paramètre significativement différents entre eux $(P=0.02)$. Pour cette variable, trois groupes ont été identifiés par le test de Newman-Keuls. L'absence de désherbage (T1) a été le moins productif avec une moyenne de 62,0 bulbes $/ 6 \mathrm{~m}^{2}$. Cependant, le désherbage permanent (T2) n'a pas été significativement différent de l'herbicide Select appliqué en post-levée (T5) avec 94,75 bulbes/ $6 \mathrm{~m}^{2}$ et 71 d'entretien de $70 \mathrm{~kg} / \mathrm{ha}$ d'urée, soit $42 \mathrm{~g} / \mathrm{planche}$ de 6 $\mathrm{m}^{2}$ est appliquée trois semaines après repiquage et une deuxième fraction de $60 \mathrm{~kg} / \mathrm{ha}$, soit $36 \mathrm{~g} /$ planche de 6 $\mathrm{m}^{2}$ un mois après la première application. L'irrigation a été implantée sur un sol argilo-sableux pour la période sèche, 2 fois/semaines du repiquage en début floraison et 2 à 3 fois par semaine à partir de la floraison.

Observations et mesures: Les observations et mesures ont porté sur les composantes du rendement, et la teneur en matière sèche des bulbes d'oignon. Le poids sec est obtenu après dessiccation des bulbes à l'étuve à $100^{\circ} \mathrm{C}$ pendant 30 heures et la teneur en matière sèche est calculée à partir de la formule suivante:

TMS $=$ (Poids sec / Poids frais) ${ }^{*} 100(\%)$

A la récolte, les nombres et les poids des bulbes récoltés, des bulbes doubles, des bulbes pourris et des bulbes de petits calibres ont été déterminés. Le nombre et le poids de bulbes vendables ont aussi été calculés. L'analyse de variance a été réalisée avec le logiciel MSAT pour étudier l'existence ou non de différences significatives $(p<0,05)$ entre les traitements. La comparaison des moyennes a été faite selon la méthode du test de STUDENT (ppds) au seuil de $5 \%$.

bulbes/ $6 \mathrm{~m}^{2}$ respectivement. Le sarclage manuel au 30ème et 60ème jour après repiquage (T3), herbicide Alligator appliqué uniquement en pré-levée (T4) ou suivi d'un sarclage manuel au 60ème jour après repiquage (T6) ne sont pas significativement différents entre eux. Le nombre total de bulbes pour ces derniers varie de 116,75 (T6) à 120 (T3) sur $6 \mathrm{~m}^{2}$ de surface de récolte.

Effets du traitement sur le poids total de bulbes récoltés: Le poids total de bulbes récoltés est présenté dans le Tableau 2. Ce paramètre a très 
significativement varié $(P=0,003)$ en fonction des différents traitements appliqués en passant chez $\mathrm{T} 1 \mathrm{de}$ $0,6 \mathrm{~kg}$ à $4,13 \mathrm{~kg}$ dans le désherbage permanent (T2). Le test de Newman-Keuls pour cette variable, a différencié 6 groupes dont le premier était constitué par
T1 (0,6 kg) bulbes, le second par T2 (4,13 kg) bulbes. Les traitements T3 $(3,17 \mathrm{~kg})$ bulbes, T4 $(2,6 \mathrm{~kg})$ bulbes, T5 0,86 kg) bulbes et T6 $(2,38 \mathrm{~kg})$ de bulbes sur $6 \mathrm{~m}^{2}$ ont représenté les troisième quatrième, cinquième et le sixième groupe respectivement.

Tableau 2 : ombre et poids total de bulbes récoltés sur $6 \mathrm{~m}^{2}$ de surface par traitement

\begin{tabular}{|c|c|c|c|c|}
\hline Traitement & $\begin{array}{c}\text { Nombre total de } \\
\text { bulbes récoltés } / 6 \mathrm{~m}^{2}\end{array}$ & $\begin{array}{l}\text { \% par rapport à T2 } \\
\text { (témoin de } \\
\text { référence) }\end{array}$ & $\begin{array}{l}\text { Poids total bulbes } \\
\qquad 16 \mathrm{~m}^{2}(\mathrm{~kg})\end{array}$ & $\begin{array}{l}\% \text { par rapport à T2 } \\
\text { (témoin de } \\
\text { référence) }\end{array}$ \\
\hline $\begin{array}{l}\text { T1 } \\
\text { T2 } \\
\text { T3 } \\
\text { T4 } \\
\text { T5 } \\
\text { T6 }\end{array}$ & $\begin{array}{c}62 \pm 5,22 \mathrm{~b} \\
94,75 \pm 12,57 \mathrm{ab} \\
120,25 \pm 4,73 \mathrm{a} \\
117,5 \pm 15,31 \mathrm{a} \\
71,75 \pm 8,61 \mathrm{ab} \\
116,75 \pm 25,4 \mathrm{a}\end{array}$ & $\begin{array}{c}-34,56 \\
- \\
+27,0 \\
+24,0 \\
-24,27 \\
+23,21\end{array}$ & $\begin{array}{c}0,60 \pm 0,23 \mathrm{c} \\
4,13 \pm 0,97 \mathrm{a} \\
3,17 \pm 0,36 \mathrm{ab} \\
2,60 \pm 0,47 \mathrm{cab} \\
0,86 \pm 0,42 \mathrm{cb} \\
2,38 \pm 0,68 \mathrm{cab}\end{array}$ & $\begin{array}{c}-85,47 \\
- \\
-23,24 \\
-37,0 \\
-79,17 \\
-42,37\end{array}$ \\
\hline & $\begin{array}{l}P=0,02 \\
F=3,31 \\
D F=5\end{array}$ & & $\begin{array}{l}P=0,003 \\
F=5,47 \\
D F=5\end{array}$ & \\
\hline
\end{tabular}

Effets du traitement sur le poids moyen unitaire bulbes: Les moyennes du poids moyen d'un fruit des différents traitements sont représentées dans le Tableau 3. La masse unitaire moyenne d'un fruit a très significativement varié pour les différents traitements $(p$ $=0,0002$ ). Le test de Newman - Keuls a différencié 4 groupes dont le premier était constitué par T1 $(5,87 \mathrm{~g})$, le second par les traitements T4 $(21,67 \mathrm{~g})$, T5 $(10,59 \mathrm{~g})$ et T6 $(19,42 \mathrm{~g})$, le troisième par T2 $(41,55 \mathrm{~g})$ et le dernier groupe est représenté par T3 (26,3 g).
Effets du traitement sur le taux de matière sèche bulbes: Le taux de matière sèche (Tableau 3 ) a très peu varié par traitement appliqué $(p=0,6)$. Le test de Newman - Keuls a observé pour ce paramètre en moyenne un seul groupe très homogène variant de $11,72 \%$ (T6) à 12,8\% (T1). Pour les traitements T2 $(12,7 \%)$, T3 $(12,73 \%)$, T4 $(12,69 \%)$ et T5 $(12,28)$, les valeurs enregistrées ont été identiques pratiquement.

Tableau 3: Poids moyen unitaire et matière sèche du bulbe par traitement

\begin{tabular}{l|c|c|c}
\hline Traitement & $\begin{array}{c}\text { Poids moyen } \\
\text { unitaire bulbe, }(\mathbf{g})\end{array}$ & $\begin{array}{c}\text { \% par rapport au T2 (désherbage } \\
\text { permanent) }\end{array}$ & $\begin{array}{c}\text { Matière sèche bulbe } \\
\text { (MS, \%) }\end{array}$ \\
\hline T1 & $5,87 \pm 3,68 \mathrm{c}$ & $-86,0$ & $12,80 \pm 1,5 \mathrm{a}$ \\
T2 & $41,55 \pm 7,48 \mathrm{a}$ & - & $12,70 \pm 4,436 \mathrm{a}$ \\
T3 & $26,30 \pm 2,58 \mathrm{~b}$ & $-37,0$ & $12,73 \pm 2,04 \mathrm{a}$ \\
T4 & $21,67 \pm 1,61 \mathrm{bc}$ & $-48,0$ & $12,69 \pm 1,79 \mathrm{a}$ \\
T5 & $10,59 \pm 4,47 \mathrm{bc}$ & $-75,0$ & $12,28 \pm 1,23 \mathrm{a}$ \\
T6 & $19,42 \pm 1,64 \mathrm{bc}$ & $-53,26$ & $11,72 \pm 2,03 \mathrm{a}$ \\
\hline & $\mathrm{P}=0,0002$ & & $\mathrm{P}=0,60$ \\
& $\mathrm{~F}=9,33$ & & $\mathrm{~F}=0,74$ \\
& $\mathrm{DF}=5$ & $\mathrm{DF}=5$ \\
\hline
\end{tabular}

Effets du traitement sur les nombres de bulbes pourris et de bulbes doubles: Les traitements appliqués, n'ont pas eu aucun impact sur le nombre de bulbes pourris récoltés sur $6 \mathrm{~m}$. Ce dernier a très peu varié par traitement $(p=0,1)$ allant de l'absence de bulbes pourris pour T1 (témoin) à 3,75 bulbes pourris lorsque l'herbicide Alligator est appliqué en pré-levée suivi d'un sarclage manuel au 60ème jour après repiquage (T6). Le test de Newman-Keuls pour cette variable a représenté un seul groupe très homogène. S'agissant des traitements restants, pour ce paramètre, les valeurs observées ont été très faibles, soit 0,25 
bulbes pourris chez $\mathrm{T} 2$ et $\mathrm{T} 3$ et pour les traitements $\mathrm{T} 4$ et T5 respectives de 0,5 et 1,75 et bulles pourris $/ 6 \mathrm{~m}^{2}$ (Tableau 4). Le nombre de bulbes doubles récolté sur 6 $\mathrm{m}^{2}$ a suivi la même tendance avec un maximum de 1 bulbe pour $\mathrm{T} 1$ et pratiquement nul pour le reste des traitements. Pour cette variable, un seul groupe homogène est constitué par le test de Newman - Keuls (Tableau 4).

Effets du traitement sur le nombre de bulbes de petits calibres (diamètre $\leq 40 \mathrm{~mm}$ ): Le nombre de bulbes de petits calibres récoltés sur $6 \mathrm{~m}^{2}$ a très peu évolué par traitement $(P=0,11)$ par traitement. Pour ce paramètre, un maximum de 26,5 bulbes a été enregistré chez T5 avec l'herbicide Select appliqué en post-levée et un minimum de 10,5 bulbes $/ 6 \mathrm{~m}^{2}$ pour le T2 avec désherbage continu et permanent. Le test de Newman-Keuls a observé pour ce paramètre en moyenne un seul groupe très homogène (Tableau 4).

Tableau 4 : Nombre de bulbes pourris, de bulbes doubles et de bulbes petits calibres par traitement

\begin{tabular}{|c|c|c|c|c|}
\hline \multirow[t]{2}{*}{ Traitement } & \multirow{2}{*}{$\begin{array}{l}\text { Nombre bulbes } \\
\text { pourris / } 6 \mathrm{~m}^{2}\end{array}$} & \multirow{2}{*}{$\begin{array}{l}\text { Nombre bulbes } \\
\text { doubles / } 6 \mathrm{~m}^{2}\end{array}$} & \multicolumn{2}{|c|}{ Bulbes de petits calibres / $6 \mathrm{~m}^{2}(\leq 40 \mathrm{~mm})$} \\
\hline & & & Nombre & $\begin{array}{l}\% \text { par rapport au nombre total } \\
\text { de bulbes récoltés } / 6 \mathrm{~m}^{2}\end{array}$ \\
\hline $\begin{array}{l}\text { T1 } \\
\text { T2 } \\
\text { T3 } \\
\text { T4 } \\
\text { T5 }\end{array}$ & $\begin{array}{l}0 \pm 0 \mathrm{a} \\
0,25 \pm 0,25 \mathrm{a} \\
0,25 \pm 0,25 \mathrm{a} \\
1,75 \pm 0,75 \mathrm{a} \\
0,5 \pm 0,28 \mathrm{a}\end{array}$ & $\begin{array}{l}0 \pm 0 a \\
0 \pm 0 a \\
0,25 \pm 0,25 a \\
0 \pm 0 a \\
0 \pm 0 a\end{array}$ & $\begin{array}{l}19,75 \pm 5,15 a \\
10,5 \pm 2,72 a \\
12 \pm 2,85 a \\
19,25 \pm 2,32 a \\
26,5 \pm 5,36 a\end{array}$ & $\begin{array}{c}31,0 \\
11,0 \\
10,0 \\
16,38 \\
36,93\end{array}$ \\
\hline \multirow[t]{2}{*}{ T6 } & $3,75 \pm 2,25 a$ & $1 \pm 0,57 a$ & $18 \pm 4,56 \mathrm{a}$ & 15,41 \\
\hline & $\begin{array}{l}P=0,10 \\
F=2,15 \\
D F=5\end{array}$ & $\begin{array}{l}P=0,07 \\
F=2,43 \\
D F=5\end{array}$ & $\begin{array}{l}P=0,11 \\
F=20,8 \\
D F=5\end{array}$ & \\
\hline
\end{tabular}

Effets du traitement sur les poids de bulbes pourris et de bulbes doubles: Le poids de bulbes pourris récoltés sur $6 \mathrm{~m}^{2}$ a très peu varié par traitement $(p=$ $0,18)$. Le test de Newman-Keuls pour cette variable a représenté un seul groupe très homogène variant de $0,01 \mathrm{~kg}$ de bulbes pour les traitements T2 et T3 à 0,05 $\mathrm{kg}$ de bulbes pourris chez le traitement (T4). L'herbicide Select en post-levée (T5) et l'herbicide Alligator appliqué en pré-levée suivi d'un Sarclage manuel au 60ème jour après repiquage (T6) ont observé respectivement $0,02 \mathrm{~kg}$ et $0,04 \mathrm{~kg} / 6 \mathrm{~m}^{2}$. Aucun bulbe pourri n'a été observé dans le témoin absolu sans (T1) sans désherbage (Tableau 5). Le poids de bulbes doubles récolté sur $6 \mathrm{~m}^{2}$ a suivi la même tendance. Seuls les traitements T6 et T3 ont enregistré des valeurs positives au demeurant très faibles respectives de $0.01 \mathrm{~kg}$ et $0,02 \mathrm{~kg}$ de bulbes doubles sur $0,6 \mathrm{~m}^{2}$. Pour le restant des traitements, ce paramètre a été nul. Pour cette variable, un seul groupe homogène est constitué par le test de Newman - Keuls (Tableau 5).
Effets du traitement sur le poids de bulbes de petits calibres (diamètre $\leq 40 \mathrm{~mm}$ ): L'analyse de ce paramètre montre une différence significative entre les traitements classés en 3 groupes selon le test de Newman-Keuls $(P=0,02)$. Les plus fortes valeurs de ce paramètre ont été observées par le Sarclage manuel au 30ème et 60ème jour après repiquage (T3) et les herbicides Alligator (en pré-levée, T4) et Select (en post-levée, T5) respectives de $0,08 \mathrm{~kg} / 6 \mathrm{~m}^{2}$ pour le premier et de $0,09 \mathrm{~kg} / 6 \mathrm{~m}^{2}$ de bulbes pour les deux derniers traitements. L'absence de désherbage (T1) et I'herbicide Alligator appliqué en pré-levée suivi d'un Sarclage manuel au 60ème jour après repiquage (T6), forment le second groupe avec chacun $0,07 \mathrm{~kg} / 6 \mathrm{~m}^{2}$ de bulbes de calibres $\leq 40 \mathrm{~mm}$. Le troisième groupe constitué par désherbage permanent et continu (T2) a enregistré $0,04 \mathrm{~kg} / 6 \mathrm{~m}^{2}$ de bulbes de calibres $\leq 40 \mathrm{~mm}$ groupe (Tableau 5). 
Tableau 5: Poids $(\mathrm{kg})$ de bulbes pourris, de bulbes doubles et de bulbes de petits calibres $(\leq 40 \mathrm{~mm})$

\begin{tabular}{|c|c|c|c|c|}
\hline \multirow[t]{2}{*}{ Traitement } & \multirow{2}{*}{$\begin{array}{l}\text { Poids bulbes pourris } \\
16 \mathrm{~m}^{2}(\mathrm{~kg})\end{array}$} & \multirow{2}{*}{$\begin{array}{l}\text { Poids bulbes doubles/ } \\
6 \mathrm{~m}^{2}(\mathrm{~kg})\end{array}$} & \multicolumn{2}{|c|}{ Bulbes de petits calibres $(\leq 40 \mathrm{~mm}$ ) } \\
\hline & & & Poids; (Kg) & $\begin{array}{l}\% \text { par rapport au poids } \\
\text { total de bulbes récoltés }\end{array}$ \\
\hline T1 & $0 \pm 0 \mathrm{a}$ & $0 \pm 0 a$ & $0,07 \pm 0,01 a b$ & 11,66 \\
\hline T2 & $0,01 \pm 0,01 a$ & $0 \pm 0 a$ & $0,04 \pm 0,007 b$ & 0,96 \\
\hline T3 & $0,01 \pm 0,01 a$ & $0.02 \pm 0.02 a$ & $0,08 \pm 0,009 a$ & 2,52 \\
\hline T4 & $0,05 \pm 0,02 a$ & $0 \pm 0 \mathrm{a}$ & $0,09 \pm 0,005 a$ & 3,46 \\
\hline T5 & $0,02 \pm 0,01 a$ & $0 \pm 0 \mathrm{a}$ & $0,09 \pm 0,004 a$ & 10,46 \\
\hline \multirow[t]{4}{*}{ T6 } & $0,04 \pm 0,02 a$ & $0,03 \pm 0,01 a$ & $0,07 \pm 0,01 a b$ & 2,94 \\
\hline & $P=0,18$ & $P=0,23$ & $\mathrm{P}=0,02$ & \\
\hline & $F=1,69$ & $F=1,50$ & $\mathrm{~F}=3,32$ & \\
\hline & $\mathrm{DF}=5$ & $\mathrm{DF}=5$ & $\mathrm{DF}=5$ & \\
\hline
\end{tabular}

Effets du traitement sur le nombre et la masse de bulbes vendables et non vendables: Le nombre de bulbes vendables récoltés sur $6 \mathrm{~m}^{2}$ a très peu varié entre les différents traitements, passant de 42,25 bulbes à 107,75 bulbes $/ 6 \mathrm{~m}^{2}$ de surface de récolte. Trois groupes ont été identifiés pour cette variable par le test de Newman-Keuls. Le premier était constitué par T3 $\left(107,75\right.$ bulbes $\left./ 6 \mathrm{~m}^{2}\right)$ avec un nombre de bulbes vendables plus élevé Les traitements T2 $(87,25$ bulbes), T3 ( 96,5 bulbes) et T6 ( 94,0 bulbes) $/ 6 \mathrm{~m}^{2}$ ont formé le second groupe avec une moyenne de 72,65 bulbes $/ 6 \mathrm{~m}^{2}$ (Tableau 7). Les traitements $\mathrm{T} 1$ et $\mathrm{T} 5$ ont constitué le dernier groupe avec 42,25 et 43,25 bulbes $/ \mathrm{m}^{2}$ respectivement. La masse de fruits vendables a fortement varié $(p<0,002)$ entre les différents traitements appliqués, passant de $0,45 \mathrm{~kg}$ (T1) à 4,07 kg/6 m² (T2). Cinq groupes ont été identifiés par le test de Newman Keuls: le meilleur était constitué par le traitement (T2) avec désherbage permanent et le dernier par le témoin (T1), sans aucun désherbage (Tableau 6). Le nombre de bulbes non vendables a peu varié $(P=0.08)$ entre 10,75 bulbes pour (T2) et 28,5 bulbes $/ 6 \mathrm{~m}^{2}$ pour T5. Un seul groupe homogène a été différencié par le test de Newman-Keuls pour cette variable (Tableau 6). Le poids de bulbes non vendables a varié $(p=0,02)$ entre $0,05 \mathrm{~kg} / 6 \mathrm{~m}^{2}$ pour T2 (désherbage permanent) et $0,14 \mathrm{~kg} / 6 \mathrm{~m}^{2}$ pour T4 (L'herbicide Alligator en pré-levée). Trois groupes ont été différenciés par le test de Newman - Keuls dont le premier était constitué par T4 $\left(0,14 \mathrm{~kg} / 6 \mathrm{~m}^{2}\right)$ et le deuxième par $T 2\left(0,05 \mathrm{~kg} / 6 \mathrm{~m}^{2}\right)$. Les traitements $\mathrm{T} 1$, T3, T5 et T6 ont formé le dernier groupe avec une moyenne de $0,1 \mathrm{~kg} / 6 \mathrm{~m}^{2}$ (Tableau 6).

Tableau 6: Nombres de bulbes vendables (NbBvd) et non vendables (NbBNvd); poids de bulbes vendables (PdBvd) et non vendables (PdBNvd) sur une surface de $6 \mathrm{~m}^{2}$

\begin{tabular}{l|l|l|l|l}
\hline Traitement & NbBvd/6 $\mathbf{~ m}^{\mathbf{2}}$ & NbBNvd/6m & PdBvd/6 $\mathbf{~ m}^{\mathbf{2}}$ & PdBNvd/6 $\mathbf{~}^{\mathbf{2}}$ \\
\hline T1 & $42,25 \pm 7,01 \mathrm{~b}$ & $19,75 \pm 5,15 \mathrm{a}$ & $0,45 \pm 0,22 \mathrm{c}$ & $0,07 \pm 0,01 \mathrm{ab}$ \\
T2 & $87,25 \pm 12,63 \mathrm{ab}$ & $10,75 \pm 2,75 \mathrm{a}$ & $4,07 \pm 0,98 \mathrm{a}$ & $0,05 \pm 0,01 \mathrm{~b}$ \\
T3 & $107,75 \pm 5,17 \mathrm{a}$ & $12,5 \pm 2,46 \mathrm{a}$ & $3,05 \pm 0,37 \mathrm{ab}$ & $0,12 \pm 0,02 \mathrm{ab}$ \\
T4 & $96,50 \pm 16,98 \mathrm{ab}$ & $21 \pm 2,48 \mathrm{a}$ & $2,48 \pm 0,47 \mathrm{cab}$ & $0,14 \pm 0,01 \mathrm{a}$ \\
T5 & $43,25 \pm 12,57 \mathrm{~b}$ & $28,5 \pm 6,98 \mathrm{a}$ & $0,75 \pm 0,41 \mathrm{cb}$ & $0,10 \pm 0,008 \mathrm{ab}$ \\
T6 & $94 \pm 27,34 \mathrm{ab}$ & $22,75 \pm 4,30 \mathrm{a}$ & $2,25 \pm 0,69 \mathrm{cab}$ & $0,12 \pm 0,02 \mathrm{ab}$ \\
\hline & $\mathrm{P}=0,02$ & $\mathrm{P}=0,08$ & $\mathrm{P}=0,002$ & $\mathrm{P}=0,02$ \\
& $\mathrm{~F}=3,40$ & $\mathrm{~F}=2,31$ & $\mathrm{~F}=5,55$ & $\mathrm{~F}=3,56$ \\
& $\mathrm{DF}=5$ & $\mathrm{DF}=5$ & $\mathrm{DF}=5$ & $\mathrm{DF}=5$ \\
\hline
\end{tabular}

\section{DISCUSSION}

Nombre récoltés de bulbes: L'analyse des composantes de rendement a monté un nombre de bulbes en général très faible par traitement pour cette étude. Cependant, les traitements avec SM (Sarclage manuel) au 30ème et 60ème jour après repiquage (T3), la Pendimethaline appliquée uniquement en pré-levée (T4) ou bien suivie d'un sarclage manuel au 60ème jour après repiquage (T6) ont produit les plus grands 
nombres de bulbes respectifs de 116,$75 ; 117,5$ et 120,25 bulbes $/ 6 \mathrm{~m}^{2}$. Au contraire de ces derniers, cette variable a été faible pour les traitements T1 $(62$ bulbes $\left./ 6 \mathrm{~m}^{2}\right)$, T2 $\left(94,75\right.$ bulbes $\left./ 6 \mathrm{~m}^{2}\right)$ et T5 $(71,75$ bulbes $\left./ 6 \mathrm{~m}^{2}\right)$. Par rapport au témoin de référence (T2) avec trois sarclo-binages effectués au cours de cycle de l'oignon, les traitements T3, T4 et T6 ont observé des gains de productivité respectifs de $27,0 \%, 24,0 \%$ et $23,21 \%$. Seuls les traitements $T 5$ et $T 1$ pour ce paramètre ont observé des valeurs respectives de $24,27 \%$ et $34,56 \%$ en dessous de celle enregistrée dans le désherbage à la demande (T2). Cependant, les nombres de bulbes enregistrés ont été plus importants que ceux obtenus dans les conditions de la fertilisation minimale de l'oignon au centre du Burkina Faso par les auteurs Garané et al. (2018a) qui ont enregistré un nombre moyen de bulbes évoluant de 37,67 bulbes/ 6

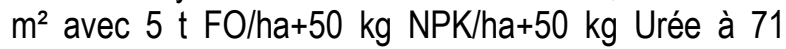
bulbes $/ 6 \mathrm{~m}^{2}$ ( $5 \mathrm{t} \mathrm{FO} / \mathrm{ha}+100 \mathrm{~kg} \mathrm{NPK} / \mathrm{ha}$ ).

Le poids moyen des bulbes: Le poids moyen des bulbes a été en général très faible par traitement. $\mathrm{Ce}$ paramètre a évolué en fonction des quantités de bulbes récoltés dans chaque traitement. Le désherbage total et permanent (T2) ou le sarclage manuel effectué

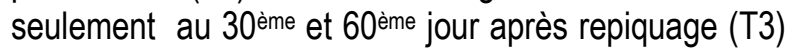
ont observé les meilleurs poids moyens bulbes respectifs de 41,55 et 26,3 g. L'herbicide Alligator appliqué seul en pré-levée (T4) ou en association avec un sarclage manuel au 60ème jour après repiquage (T6) ont pour ce paramètre enregistré des valeurs deux fois plus faibles et presque identiques respectives de $21,67 \mathrm{~g}$ et $19,42 \mathrm{~g}$. Seuls les traitements témoin $(5,87$ $\mathrm{g})$ et la Clethodime appliquée en post-levée $(10,59 \mathrm{~g})$ ont observée pour cette variable des valeurs plus faibles. Par rapport au témoin de référence (T2), les traitements T6, T5 et T1 (témoin) ont observé pour ce paramètre des chutes très importantes respectives de $53,26 \% ; 75,0 \%$ et $86,0 \%$. S'agissant des traitements T3 (sarclage manuel au 30ème et 60ème jour après repiquage) et T4 (Alligator appliqué en pré-levée), ces pertes bien que élevées ont été relativement moins importantes respectives de $37,0 \%$ et $48,8 \%$. Indépendamment des traitements appliqués, le Violet de Galmi a observé un poids moyen bulbe faible compris entre 5,67 g (T1) et 41,55 g (T2). De même, Pour ce paramètre, AVRDC (2013) en culture de saison sèche et fraiche de novembre (2012) à avril (2013) a été obtenu un résultat quelque peu meilleur de $21,7 \mathrm{~g}$ pour cette variété qui en temps normal de production atteint un poids de $150 \mathrm{~g}$ (Assogba-Komlan et al, 2007 AVRDC, 2014). Au cours de la saison d'hivernale,
Assogba-Komlan et al. (2007) ont enregistré des bulbes de poids moyen très faibles respectifs de $9,1 \mathrm{~g}$ et $7,5 \mathrm{~g}$. Ces résultats médiocres de ce cultivar pendant cette période ne peuvent s'expliquer que par son non adaptation à la culture d'hivernage.

Les rendements bulbes: Les poids des bulbes récoltés sur $6 \mathrm{~m}^{2}$ ont été en générale faibles par traitement de l'essai. Cependant, les rendements bulbes observés ont été quelques peu meilleurs pour le désherbage permanent $\left(4,13 \mathrm{~kg} / 6 \mathrm{~m}^{2}\right)$ et dans une moindre mesure dans le traitement T3 $\left(3,17 \mathrm{~kg} / 6 \mathrm{~m}^{2}\right)$ avec sarclage manuel effectué au 30ème et 60 ème jour après repiquage. L'absence de désherbage (T1) et l'herbicide Select en post-levée (T5) ont entrainé une plus faible masse de bulbes à la récolte. Une comparaison des différents traitements par rapport au témoin de référence (T2) a montré des pertes de productivité allant de - 23\% (T3) à - 85,47\% (T1). Pour les traitements $\mathrm{T} 4, \mathrm{~T} 6$ et $\mathrm{T} 6$, les pertes de rendements ont été importantes respectives de - 37\%, - 42,37\% et $79,17 \%$. Des tests variétaux de saison sèche et fraiche conduits par AVRDC (2013) de novembre (2012) à avril (2013) ont permis d'obtenir un rendement bulbes comparable pour la variété violet de Galmi de 19,6 t/ha $\left(3,06 \mathrm{~kg} / 6 \mathrm{~m}^{2}\right)$. La période de culture pourrait expliquer ces meilleurs résultats dans la mesure où ce dernier a été caractérisé par un résultat très médiocre de 1,9 t/ha observé en culture hivernale (Garané et al; 2018b). Le rendement très médiocre du violet de Galmi ne peut s'expliquer que par la non adaptabilité de cette variété à la culture d'hivernage. Cette dernière après sa sélection présente un rendement moyen de 45 t/ha pouvant culminer à 60 t/ha, (Assane Dagna, 2006). Pour cette variété, les conditions de fertilisation ont un impact sur son rendement. Aussi, Garané et al. (2018a), dans les conditions de fertilisation réduite, ont obtenu des rendements de $2,16 \mathrm{~kg} / 6 \mathrm{~m}^{2}$ bulbes pour ( 5 t FO/ha+50 kg NPK/ha+50 kg Urée) et de $2,8 \mathrm{~kg} / 6 \mathrm{~m}^{2}$, $2,55 \mathrm{~kg} / 6 \mathrm{~m}^{2}$ et $2,51 \mathrm{~kg} / 6 \mathrm{~m}^{2}$ pour les traitements $(5 \mathrm{t}$ FO /ha+100 kg NPK/ha), (20 t FO/ha+50 kg NPK/ha) et (5 t FO/ha+50 kg Urée/ha)respectivement. Ces rendements ont été en général très faibles pour cette variété qui en temps normal de production atteint dans sa sélection un rendement moyen de 45 tha pouvant culminer à 60 tha (Assane Dagna, 2006). Le sarclobinage répétitif aussi bien dans le désherbage total et permanent que dans le sarclage manuel au 30ème et 60 ème jour après repiquage en plus d'améliorer l'état phytosanitaire de la parcelle par la destruction des mauvaises herbes contribue a éviter le tassement et la compaction des sols et l'érosion (Boiffin et al., 2001; 
Chervet et al., 2005; Mrabet, 2010) qui s'accompagne d'une réduction de la porosité du sol, de la circulation de l'oxygène et d'une augmentation du taux de saturation de la porosité en eau (Köller, 2003; Labreuche et al., 2007). Ces changements limitent le développement et l'activité des microorganismes aérobies et réduisent ainsi la minéralisation de la matière organique et de l'azote organique (Young et Ritz, 2000; Pekrun et al., 2003; Grigera et al., 2007), principal élément nutritif pour les végétaux. (Lipiec et Stepniewski, 1995). De façon générale, de désherbage en culture maraîchère est effectué grâce aux outils manuels et instruments attelés à des animaux avec cependant une prédominance du désherbage manuel (Gill 1979, 1980). Or ce type de désherbage en cultures maraîchères exige une main-d'œuvre importante qui n'est pas toujours disponible et un nombre élevé d'heure de travail. Ainsi Moody cité par Holm et al. (1976) signal l'exemple de trois villages du Nigéria où les agriculteurs consacrent respectivement 74,61 et 56 pour cent de leur temps de travail au désherbage. Le contrôle chimique des mauvaises herbes par des herbicides sélectifs (De Linan, 2000; Arsenovic et Kunkel 2001; Chikoye et al. 2002) bien que mineur, demeure indispensable pour les grandes superficies quand la main d'œuvre est rare enfin de minimiser les dépenses et réduire la charge de travail (Marmotte, 2000). Son association à des sarclo-buttages fréquents (William et al. 2000) mérite une attention particulière car la gestion des mauvaises herbes en cultures maraîchères est une question sensible où l'on doit éviter les problèmes écologiques dans des rotations qui sont souvent très courtes et intensives favorisant l'impact de la toxicité des herbicides sur la culture suivante lorsque le cycle de culture précédente est assez court (Labrada, 2005). Un contrôle biologique des mauvaises herbes en culture maraîchères par des insectes phytophages (Klein, 2011) constitue une voie dans la perspective d'une agriculture écologique et biologique à envisager.

Matière sèche des bulbes: Le taux de matière sèche a très peu varié par traitement appliqué. Le test de Newman - Keuls a observé pour ce paramètre en moyenne un seul groupe très homogène variant de $11,72 \%$ (T6) à 12,8\% (T1). Pour les traitements T2 $(12,7 \%)$, T3 $(12,73 \%)$, T4 $(12,69 \%)$ et T5 $(12,28 \%)$, les valeurs enregistrées ont été identiques pratiquement. Des études antérieures ont rapporté que le taux de matière sèche des bulbes varient normalement selon les variétés entre $7 \%$ et $18 \%$ du poids frais (Abhayawick et al., 2002). De plus, ces résultats corroborent avec ceux trouvés dans la littérature (Currah \& Proctor; 1993) qui soutiennent que le taux de matière sèche varie entre des niveaux assez bas (7 à $10 \%$ ) et des niveaux plus élevés ( 15 à $20 \%$ ). Le taux de matière sèche mérite également une attention particulière du point de vue de l'aptitude à la conservation (Silué et al., 2003; Assogba-Komlan et al, 2007; Konaté et al., 2017). Les oignons riches en matière sèche sont plus fermes et de ce fait plus résistants aux lésions causées par le transport et la manipulation. Mais ce sont des oignons qui produisent des rendements moins élevés (Michel et Claude, 2003). De plus, la variété est connue pour sa bonne conservation qui est de l'ordre de $91 \%$ en 3 mois et $84 \%$ en 6 mois (Mémento de l'agronome, 2002; Assane Dagna, 2006).

Le nombre et le poids de bulbes de petits calibres (diamètre $\leq \mathbf{4 0} \mathrm{mm}$ ): Ce paramètre est aussi bien important pour le rendement que pour la qualité des oignons à la récolté. Par traitement, le nombre de bulbes de calibres inférieurs $\leq 40 \mathrm{~mm}$ récoltés sur $6 \mathrm{~m}^{2}$ a très peu évolué. Pour ce paramètre, un maximum de 26,5 bulbes a été enregistré chez $T 1$, témoin sans désherbage et un minimum de 10,5 bulbes $/ 6 \mathrm{~m}^{2}$ pour T2 avec désherbage continu et permanent. Par rapport au nombre total de bulbes récoltés sur $6 \mathrm{~m}^{2}$, l'absence de désherbage (T1) et L'herbicide Select appliqué en post-levée (T5), ont eu un effet plus négatif sur ce paramètre avec les plus forts taux respectifs de 31,0\% et $36,93 \%$. L'herbicide Alligator appliqué uniquement en pré-levée (T4) ou suivi d'un sarclage manuel au 60ème jour après repiquage (T6) ont formé le second groupe avec des taux respectifs de $15,41 \%$ et $16,38 \%$ de bulbes de petits calibres. Le désherbage total et permanent (témoin de référence) et le sarclage manuel au 30ème et 60ème jour après repiquage (T3) ont observé les plus faibles valeurs de ce paramètre avec seulement $11,0 \%$ et $10,0 \%$ de taux respectivement. Ces deux derniers traitements ont entrainé un effet positif plus important sur cette variable. La présence plus ou moins importante de bulbes de petits calibres (10\%-37\%) confirme la faible performance générale de la variété et explique les faibles rendements observés des différents traitements. Les traitements appliqués ont influencé les poids enregistrés des bulbes de petits calibres. Aussi, pour cette variable, les traitements T3, T4 et T5 ont observé les plus fortes valeurs respectives de $0,09 \mathrm{~kg} / 6 \mathrm{~m}^{2}$ pour le premier et de $0,08 \mathrm{~kg} / 6 \mathrm{~m}^{2}$ pour les deux derniers. Les traitements T1 et T6 ont formé le second groupe avec tout deux $0,07 \mathrm{~kg} / 6 \mathrm{~m}^{2}$, tandis que T2 constituait le dernier groupe avec le plus faible poids 
de bulbes de petits calibres de $0,04 \mathrm{~kg} / 6 \mathrm{~m}^{2}$. Comparativement au poids total de bulbes récoltés par traitement, l'absence de désherbage (T1) et l'herbicide Select appliqué en post-levée (T5) ont eu un effet plus négatif sur ce paramètre. Les bulbes de petits calibres ont constitué $11,66 \%$ et $10,46 \%$ du poids total bulbes respectivement. S'agissant des autres traitements, ce paramètre de $0,96 \%$ a été très faible chez $\mathrm{T} 2$, tandis que pour T3, T4 et T6 il était représentait seulement $2,52 \%, 3,46 \%$ et $2,94 \%$ du poids total bulbes récoltés respectivement. De façon générale le nombre de bulbes de calibres inférieurs à $40 \mathrm{~mm}$ a été inférieur a $40 \%$ pour l'ensemble des traitements de l'essai. Comparativement a l'ensemble des bulbes récoltés, cette classe de bulbes a varié de 10\% (T1) à 36,38\% (T5); soit $62,63 \%$ à $90 \%$ de bulbes de calibres supérieur à $40 \mathrm{~mm}$ suivant le traitement. Indépendamment des traitements appliqués, ce résultat pour le Violet de Galmi reste très positif pour la période de culture dans la mesure où sur certain site d'étude le violet de Galmi, oignon non destiné à la culture d'hivernage est reconnu comme une variété produisant des bulbes de calibre moyen (40-60 mm). Par ailleurs, la sélection du matériel végétal et les techniques culturales sont menées afin d'obtenir des bulbes 40-60 $\mathrm{mm}$ pour le Violet de Galmi en saison normale de culture (N'Guessan, 1997). La prédominance de bulbes de calibres supérieurs à $40 \mathrm{~mm}$ peut être un critère déterminant dans l'appréciation générale de la qualité de la récolte. Les oignons de très faibles calibres sont en générale dépréciée par le marcher et n'ont pas une grande valeur commerciale

Le nombre et le poids de bulbes vendables: Les traitements appliqués ont impacté sur le nombre de bulbes vendables récoltés avec un minimum de 42,25 bulbes $/ 6 \mathrm{~m}^{2}$ avec le témoin absolu (T1) et un maximum de 120,25 bulbes $/ 6 \mathrm{~m}^{2}$ dans Sarclage manuel au 30 ème et 60 ème jour après repiquage (T3). Hormis ce dernier, l'herbicide Alligator appliqué en pré-levée seul

\section{CONCLUSION ET APPLICATION DES RESULTATS}

Le Sarclage manuel au 30ème et 60ème jour après repiquage (T3), la Pendimethaline appliquée uniquement en pré-levée (T4) ou suivie d'un Sarclage manuel) au 60ème jour après repiquage (T6) ont produit les plus grands nombres de bulbes récoltés respectifs de 116,$75 ; 117,5$ et 120,25 bulbes $/ 6 \mathrm{~m}^{2}$. Le poids moyen bulbes récoltés a été plus performant avec le désherbage total et permanent (T2) et le sarclage manuel effectué au 30ème et 60ème jour après repiquage (T3) avec 41,55 et $26,3 \mathrm{~g}$
(T4) ou suivi d'un Sarclage manuel au 60ème jour après repiquage (T6) ont aussi observé des valeurs importantes pour cette variable respectives de 117,5 et 116,75 bulbes $/ 6 \mathrm{~m}^{2}$. Comparativement au nombre total de bulbes récoltés, le pourcentage de bulbes vendables a été très important pour l'ensemble de l'essai et a varié de $60,27 \%$ (T5) à $92,08 \%$ avec le témoin de référence (T2). Pour le témoin absolu, ce taux a été de $68,14 \%$, tandis que les traitements restants $\mathrm{T} 3, \mathrm{~T} 4$ et T6 avaient par rapport au nombre total de bulbes $89,6 \%, 82,14 \%$ et $80,51 \%$ de bulbes vendables respectivement. Masse de bulbes vendables a fortement varié entre les différents traitements appliqués, passant de 0,45 kg (T1) à 4,07 kg/6 m² (T2). Le désherbage total et permanent (T2) et le Sarclage manuel au 30ème et 60ème jour après repiquage (T3) ont observé les meilleurs performances respectives de $4,07 \mathrm{~kg}$ et $3,05 \mathrm{~kg} / 6 \mathrm{~m}^{2}$. Les traitements T4 et T6, ont enregistré $2,48 \mathrm{Kg}$ et $2,25 \mathrm{~kg} / 6 \mathrm{~m}^{2}$ respectivement. L'absence de désherbage (T1) ou l'herbicide Select en post-levée (T5) ont été moins performants pour ce paramètre avec $0,45 \mathrm{~kg}$ et $0,75 \mathrm{~kg} / 6 \mathrm{~m}^{2}$ enregistrés respectivement. Une comparaison à la masse totale de bulbes récoltés par traitement a montrée que les pourcentages de bulbes vendables ont été très importants par traitement. Si dans le traitement Témoin, $75 \%$ de la masse tale des bulbes ont été vendables, ce taux a cru pour atteindre $98,54 \%$ avec le désherbage permanent et continu (T2), soit, presque la totalité des bulbes récoltés. Hormis le témoin absolu (T1), seule la Clethodime en post-levée (T5) a observé pour ce paramètre une valeur inférieure a $90 \%$, soit $87,2 \%$. Le sarclage manuel au 30ème et 60 ème jour après repiquage (T3) et la Pendimethaline appliquée uniquement en prélevée (T4) ou suivi d'un Sarclage manuel au 60ème jour après repiquage (T6), ont observé pour cette variable des valeurs très importantes respectives de $96,21 \%, 95,38 \%$ et $94,53 \%$.

respectivement. La plus faible valeur de $5,87 \mathrm{~g}$ pour cette variable a été enregistrée avec le témoin absolu (T1). Les rendements bulbes sur $6 \mathrm{~m}^{2}$ ont été quelques peu meilleurs dans le désherbage permanent $(4,13$ $\mathrm{kg} / 6 \mathrm{~m}^{2}$ ) et dans une moindre mesure lorsque le sarclage manuel est effectué au $30^{\text {ème }}$ et $60^{\text {ème jour }}$ après repiquage $\left(3,17 \mathrm{~kg} / 6 \mathrm{~m}^{2}\right)$. L'absence de désherbage (T1) ou encore l'herbicide Select appliqué en post-levée (T5) ont observé les plus faibles valeurs pour ce paramètre. Par traitement, le nombre de bulbes 
de petits calibres (diamètre $\leq 40 \mathrm{~mm}$ ) a observé un maximum de 26,5 bulbes chez $\mathrm{T} 5$ et un minimum de 10,5 bulbes $/ 6 \mathrm{~m}^{2}$ pour le T2 Par contre, pour ce même paramètre, les poids observés ont été observées plus importants avec le Sarclage manuel au 30ème et 60ème jour après repiquage $\left(0,08 \mathrm{~kg} / 6 \mathrm{~m}^{2}\right)$ et les herbicides Alligator en pré-levée $(0,09 \mathrm{~kg} / 6 \mathrm{~m})$ et Select en postlevée $\left(0,09 \mathrm{~kg} / 6 \mathrm{~m}^{2}\right)$. Le sarclage manuel effectué seulement au 30ème et 60ème jour après repiquage (T3) a formé le plus nombre de bulbes vendables

\section{REFERENCES}

Abhayawick L., Laguerre J.C., Tauzin V. and Duquenoy A. 2002. Physical properties of three onion varieties as affected by the moisture content. Journal of Food Engineering, 55: 253-262.

Alessandro S. and Alseny S. 2008. Note technique: Évaluation sous-régionale de la chaine de valeurs oignon échalote en Afrique de l'ouest, ATP, p. 58

Arsenovic M. et Kunkel, D. L. 2001. The IRA-4 projet. National Agricultural Program for pest management solution, EWRS working group, meeting on weed management systems in vegetables, Zaragoza, Spain. P. 1.

Assane Dagna M. 2006. Les effets de la réappropriation de la culture du Violet de Galmi par les producteurs d'oignon de la région de Tahoua-NIGER, sur la dynamique du territoire local, l'organisation sociale et économique, Thèse de doctorat. Université de Toulouse-Le. Option développement rural, p. 281.

Assogba-Komlan F.V., Bello S. et Baco M.N. 2007. Sélection participative au champ de quelque cultivars d'oignon de jours courts pour le département de l'Alibori. Bulletin de la Recherch Agronomique du Bénin, 58:45-55.

AVRDC (The World Vegetable Center) 2014. Year in Review 2013. Develop short-day red onions and yellow onions for improved yield, extended shelf-life, and/or Stemphylium resistance, p.78.

Bello S. 2012. Analyse diagnostique de la production et de la commercialisation de l'oignon de 1995 à 2009 au Nord-Est du Bénin. Accepté par BRAB en juillet 2012, INRAB, Bénin, p. 35.

Bello S., Assogba-Komlan K.F. et BAC M.N. 2004. Guide pour la production de l'oignon dans l'Alibori. INRAB-CRAN/AFD/PADSE, Bénin, p. 53. $\left(107,75\right.$ bulbes $\left./ 6 m^{2}\right)$. Aucune différence n'a été constaté entre les traitements T2 $\left(87,25\right.$ bulbes $\left./ 6 \mathrm{~m}^{2}\right)$, T3 $\left(96,5\right.$ bulbes $\left./ 6 \mathrm{~m}^{2}\right)$ et T6 $\left(94,0\right.$ bulbes $\left./ 6 \mathrm{~m}^{2}\right)$; tandis que la masse de bulbes vendables a fortement varié passant de $0,45 \mathrm{~kg}$ (T1) à $4,07 \mathrm{~kg} / 6 \mathrm{~m}^{2}$ (T2). L'association des sarclo-buttages fréquents avec des herbicides sélectifs (T6) reste envisageable dans la gestion des mauvaises herbes sur les grandes superficies en vue d'une réduction des coûts d'exploitation en cultures maraichères.

Boiffin J., Malezieux E. \& Picard D. 2001. Cropping systems for the future. In Nösberger J. Geiger, H.H., Struik, P. C. (eds.), Crop Science, Pp. 261-280.

Carlson H.L. and Kirby D. 2005. Effect of Herbicide Rate and Application Timing on Weed Control in Dehydrator Onions. University of Florida, Intermountain Research and Extension Center, 4 (2005) 115p. Qasem Jr: 2006. Chemical. Weed Control in Seedbed Sown Onion (Allium cepa L.). Crop Prot., 25: 618622.

Chervet A., Ramseier L., Sturny W.G. \& Tschannen S. 2005. Comparaison du semis direct et du labour pendant 10 ans. Revue suisse Agric. 37 (6): 249-256.

Chikoye D., Monyong V.M. Carski R. Ekelene F., Gbehounou G. and Abanchede A. 2002. Reponse of speargrass (Imperata cylindrical) to cover crops integrated with hand weeding and chemical control in maize and cassava. Crop protection, 21: 145-156

Currah L. \& Proctor F.J. 1993. La culture et la conservation des oignons sous les tropiques. CTA/NRI, p. 161.

De Linan G. 2002. Vademecum de productos fitosanitarios y nutricionales. $18 \mathrm{ed}$. agrotecnicas S.L. Madrid. PP. 196-275.

DGPSA (Direction Générale des Prévisions et des Statistiques Agricoles). 2008. Rapport analyse de la filière maraichage. Ouagadougou, Burkina Faso, p. 117.

DPSAA (Direction de la prospective et des statistiques agricoles et alimentaires). 2011. Rapport d'analyse du module maraîchage. Ouagadougou, Burkina Faso, p. 214.

Garané A., Some K.,Yonli D., Traore M., Sawadogo M et Nikiema J. 2018a. Effets de la fertilisation minimale de l'oignon (Allium cepa L.) sur son 
enherbement et le rendement bulbes au Centre du Burkina Faso. Afrique Science, 14(4) $40-54$.

Garané A., Some K., Nikiema J., Traore M., Sawadogo M. et Belem J. 2018b. Evaluation du comportement au champ de quelques variétés d'oignon (Allium cepa L.) et d'échalote (Allium cepa var. asculoni cum) pour la culture d'hivernage au centre du Burkina Faso. Int. J. Biol. Chem. Sci., 12(4): 1836-1850.

Gill, H.S. 1979. Annual Report of the Departement of Agronomy, 1978-1979. Punjab Agricultural University, Ludhiana, India.

Gill, H.S. 1980. Annual Report of the Department of Agronomy, 1979-1980. Punjab Agricultural University, Ludhiana, India.

Grigera M.S., Drijber R.A. et Wienhold B.J. 2007. Redistribution of crop residues during row cultivation creates a biologically enhanced environment for soil microorganisms. Soil and Tillage Research 94:550-554.

Hodonou-Gotoechan H. et Savi A.D. 2001. Etude diagnostique sur la production de l'oignon dans le département de l'Alibori. Actes de l'Atelier Scientifique Nord Edition 2001.Parakou. Bénin, Pp.109-119. http://www.slire.net/download/757/article5_bra b58_assogbakomlan_et_al_s_lection_participa tive_au_champ-cultivars-oignon.pdf

Holm, Leroi G. 1976. The important of weeds in world food programmes proceedings. British Crop protection conference. Weeds, 3: 733-769.

Klein, H. African Entomologies. 2011, 13 (2): 515 519.

Köller K. 2003. "Techniques of Soil Tillage", In A. El Titi, ed. Soil Tillage in Agro écosystème CRC Press LLC, Boca Raton, Pp. 1-25.

Konate M., Parkouda C., Tarpaga V., Guira F., Rouamba A. et Sawadogo H. 2017. Evaluation des potentialités nutritives et l'aptitude à la conservation de onze variétés d'oignon (Allium cepa L.) bulbe introduites au Burkina Faso Int. J. Biol. Chem. Sci., 11(5):2005-2015.

Labrada, R. 2005. Gestion des mauvaises herbes pour les pays en developpement. Author FAO, Rome, AGP. Publsher, FAO, Rome (Italy), pp 151-164.

Labreuche J., Viloingt T., Caboulet D., Daouze J.P., Duval R., Ganteil A., Jouy L., Quere L., Boizard H. et Roger-Estrade J. 2007. "Evaluation des impacts environnementaux des Techniques Culturales Sans labour (TCSL) en France". Partie I: La pratique des TCSL en France, Ademe.

Lipiec J. et Stepniewski W. 1995. Effects of soil compaction and tillage systems on uptake and lose of nutriments. Soil \& Tillage Research, $35: 37-52$.

Mario. L. 2015. Utilisation des herbicides dans l'oignon semé en sol organique, 25-08-2015 Agriculture, Pêcherie et Alimentation.

Marmotte P. 2000. La gestion de l'enherbement et l'emploi des herbicides dans les systèmes de culture en zone soudano sahélien en Afrique de l'Ouest et du Centre. Formation du CIRAD - CA- GEC-AMATROP. pp. 11- 16.

Mémento de l'agronome 2002.

Michel P, Claude F. 2003. Histoires de légumes: Des origines à l'orée du XXle siècle Science. INRA, Paris, ISBN:978-2-7380-1066-7.

Mrabet R. 2010. Climate change and carbon sequestration in the Mediterranean basin: contribution of no tillage systems. Les actes des 4e Rencontres Méditerranéennes du Semis Direct. Sétif Algérie 3, 4,5 mai 2010. N spécial revue Recherche agronomique. Pp. 106-126.

N'Guessan K.J. 1997. Atelier de formation sur la filière semences oignon, la production des semences d'oignon et le contrôle de qualité des semences. Ferké, les 14,15 et 16 octobre 1997, 10p.

Pekrun C., Kaul H.P. et Claupein W. 2003. Soil tillage for sustainable nutrient management. In El Titi, A. (ed.). Soil tillage in agroecosystems, CRC Press, New-York (USA), pp. 83 -113.

Silué S., Fondio L., Coulibaly M.Y. and Magein H. 2003. Sélection de variétés d'oignon (Allium cepa L.) adaptées au nord de la Côte d'lvoire. Tropicultura, 21(3):129-134.

Smith R. \& Fennimore S.A. 2008. Management guidelines: Onion and Garlic weeds" and Natural Resources. M L Flint, Ed University of California Publication, pp 34-53.

Son G., Kiogo R, Yé SG. 2016. Analyse des systèmes de production de l'oignon bulbe autour du barrage de Goinré dans la province du Yatenga au Nord du Burkina Faso. Int. J Biol. Chem. Sci., 10(3): 1173-1183,

Thiombiano A: 2005. "Les combretaceae du Burkina Faso: taxonomie, écologie, dynamique et régénération des espèces". Thèse de Doctorat 
d'État ès Sciences Naturelles, Université de Ouagadougou, p. 290.

Young I.M. et Ritz K. 2000.Tillage, habitat space and function of soil microbes. Soil and Tillage. Research, 53:201-213.
William R.D., Ball T., W. Morishita D.W. et Hutchinson P. 2000. Weed management in vegetable crops. Pacific Northwest weed control handbook. Extension services of Orogon St. Univ., Washinton State Univ. and Univ. of Idaho. USA. pp. 244-274. 